\title{
On Gender Justice in School Education
}

\author{
Ruohong Zhang \\ College of Letters and Science, University of California, Davis 95616, USA, rhgzhang@ucdavis.edu
}

Keywords: School education; Gender justice; Research analysis

\begin{abstract}
In her masterpiece, The Second Sex, French writer Simone de Beauvoir stated: "Human society is devoid of all natural elements. Females, like many others, are a product of civilization. "Other people's intervention in her fate was usually decisive. If we had acted in a different direction, a totally different outcome would have resulted. The status of women has improved with the times, but the existence of sexual injustice cannot be denied. Overt gender discrimination has decreased considerably, but gender injustice is not currently decreasing and is everywhere in schooling.
\end{abstract}

\section{The Implications of Gender Justice}

Gender justice is often associated with the Western feminist and women's movements. Western feminist theory is complex, with many schools of thought and different concerns, making it difficult to define gender justice correctly. Gender equality includes respect for difference and freedom of choice, with gender justice as its primary component. In general, the importance of gender equality lies in the values of men and women. Throughout human history, there has always been an inequality of values between men and women, with men in a position of exalted leadership. These views have maintained the hegemony of men for thousands of years and have unconditionally weakened the position of women. If we want to find the basis for gender equality, men and women tend to focus on biological differences, with the aim of identifying the physical differences between men and women that are linked to the social division of labour and lead to differences in social status. But we must not distort it; as a gift from the Creator, it makes no difference in terms of social values. Women cannot, therefore, be excluded from certain areas of life.

We should embrace gender equality without any gender barriers or limitations, starting with the requirement of gender equality, whether in the sphere of human activity, the public sphere, the individual psyche, or the psycho-social sphere. This is what we commonly refer to as the "infinite field," which is yet another requirement for respecting differences and gender equality. Gender equality advocates believe that there are biological and socio-cultural differences between the sexes, and that it is incorrect to believe that inequality between men and women is caused by arbitrary socio-cultural and random differences in natural resources. There is a legitimate basis for gender equality: men and women are born differently; demands for gender equality must be justified; and demands and proposals for gender equality aim to eliminate differences. Their demands and claims to legitimacy are not for equality in life, but for equality between men and women, who have different physical conditions. This is why we should respect gender differences in two ways, namely in biological differences and in sociocultural gender differences.

We know that "sex" and "gender" are different concepts. "Sex" refers to the anatomical and physical characteristics of males and females, the biological nature of human beings. "Gender" refers to psychological and social aspects, cultural differences and social well-being. We were not born with a gender, although we are born with individuality. Socially acknowledged male and female characteristics are acquired through subtle learning. If they accumulate over time, they are considered to be natural phenomena. With regard to the social differences between men and women, we should focus on the historical and cultural factors behind the differences, which are the result of historical and cultural influences.

These characteristics are socio-cultural in nature, based on the requirements of sexual justice. We must respect the compensation and welfare of women in a given historical period, particularly those in vulnerable positions such as childbirth and domestic work, which allows both sexes to access social welfare and freely choose. In social and cultural history, men and women are expressions of humanity and form. This is why men and women have different personalities, and women can concentrate the best qualities of other people in themselves. As a result, deeper sexual technology promotes education and, more specifically, equal opportunities for women to choose their own personalities and, more specifically, to realize female ideals and persecution.

In other words, biology does not determine the appropriateness of gender characteristics; each person chooses between purely female and purely male attributes in order to maximize their humanity. The essence of "male-female communion" is not to force a person to be female or male, nor to be both male and female, but to express all the humanity of their biological sex while denying the right to free choice. There is no distinct dividing line between the sexes. It is not a skill that men and women can learn to suit their needs. As a result, sexual justice should provide all options. For example, women should eliminate the biological need to do things, such as the fact that being the CEO of a company is considered 
a male prerogative. When women get involved, they become 'strong women', which is a form of sexual injustice. There are no physical requirements.

Opportunities should be holistic, ensuring the participation of all, including women, and that women's free choice and development should not be forced or excluded by perceptions and institutions. It is clear from the preceding that sexual contribution to emotions necessitates not only personality equality and equal opportunities for men and women, but also personality freedom. In exceptional circumstances, special consideration should be given to compensating vulnerable groups.

\section{Gender Injustice in School Education}

As Fouad points out in "The Second Sex," women's status has improved significantly in many ways. However, it is undeniable that the consequences of long-standing Southern dominance are irreversible, that women continue to be marginalized, and that sexual injustice is pervasive. Unconstrained by law, the Aristotelian tradition posited the man as a positive factor and the woman as a negative one, and this argument spread widely. School rules not only reflect gender inequality, but they also exacerbate it, primarily in two areas: the curriculum and teachers.

\subsection{Gender Injustice in the Curriculum}

Courses are typically classified as explicit or implicit. Gender bias is communicated through professional teaching in the interpretive curriculum. The interpretive curriculum has the potential to influence all aspects of students' daily school lives. Subject and content bias are examples of gender bias in the explicit curriculum. Many people think about women in the abstract when discussing this topic. I believe that women's spatial imagination is inferior to men's [1], that some subjects, such as mathematics and chemistry, are unsuitable for women, and that women believe they can excel in these subjects, leading people to wonder if they will lose confidence in studying the humanities and social sciences.

The spiritual sciences have a lower status than the natural sciences, merely read. Many experiments, like those in natural sciences, demonstrate this without a logical basis. According to this viewpoint, women's status has once again declined, and this gender bias is even more visible in terms of subject matter and content. Several studies of Chinese textbooks and extracurricular reading material show that men are preferred over women in various textbooks in a sizable proportion. When thinking of history books, we see very few women, with most of they are buried beneath a sea of men. As a result, male have long mastered the period of history. It's also difficult to explain in other modern textbooks where men outnumber women.

Furthermore, the image of women in textbooks is relatively uniform and limited to specific behaviors. It reinforces societal gender roles such as teachers, housewives, and managers, which is the result of stereotypes, with men playing a relatively rich and varied role. Essentially, it is more severe in a variety of fields, particularly in the evaluation of highlevel professions such as leaders and scientists [2]. Some textbooks even portray women as short-sighted, superficial, weak and cowardly. They are portrayed as anxious people, in the sense that pupils learn primarily about male experiences and achievements, learning in textbooks about male excellence while knowing little about female achievements, and women end up constituting a prejudice whose internal processes continue to exist in schools and in the lives of pupils because it covers all aspects of learning and has a more serious impact on them.

In terms of staff structure, headmasters are male, while specifiers and teachers are female, especially in primary and secondary schools where psychological support is provided to students; that is, women have a low status and are in a subordinate position suitable for support and leadership work, whereas men have a high status and are suitable for guidance and direction. This is a typical and reasonable professional request. The second point to mention is the masculinity of school facilities. On the surface, there appear to be no distinctions between students, and the same training is provided to the same teacher and friend in the same school. They carry out their activities in the same location, but the most visible example of the school's soft infrastructure is that the school uniforms are not designed with women in mind, but they should choose casual sportswear for men. Third, the male approach to school teaching is undeniable. There are distinctions between male and female students' cognitive styles which can not be divided as advantages or disadvantages, both sides approaching learning differently.

It is widely assumed that women excel at collaboration, narrative and interaction, men excel at solitude,abstract thinking and reflection. However, competitive teaching methods appropriate for boys are widely accepted in the classroom, and the expression of teaching attitudes is how boys improve. Girls are at a disadvantage when it comes to learning. Gender equality is implied by language in everyday communication. Language serves as a sociocultural medium while gender bias in society and culture is frequently unspoken language, which have gradually gained acceptance, becoming a universal language. Much of the language that affects, stigmatizes, struggles with, and satirizes women diminishes and stigmatizes them. Many of the words associated with masculinity, a language with gender-specific effects, are ingrained in children's minds and are reflected in communication. Some boys who do not conform to social expectations greet their coworkers with "the same accent as women" and the superiority of other men, with no regard for differences or boundaries.

\subsection{Gender Injustice Revealed by Teachers' Educational Behavior}


Teachers, as subjects of social culture, are heavily influenced by it. As a result, gender bias is unavoidable, developing during the conscious or unconscious educational process when communicating with students. Male and female students receive different levels of attention from teachers, and certain common behaviors are more likely to be repeated. Teachers, for example, address boys more clearly than girls, focusing on them more, and ask probing questions about boys' presentations. The teacher allotted boys more time and emphasized verbal and nonverbal responses [3]. In the classroom, the teacher encourages boys to respond or comment more. This is unconscious and social behavior on the part of the language teachers. While this may be the result of long-term cultural influences, teachers' behavior reinforces sexual injustice and passes it on to the next generation as a pillar of social culture. Gender equality is reflected in the organization and language of instruction, which benefits men while harming women. Sexual injustice pervades society. It transforms into a continuous thread that is amplified and extended.

\section{Conclusion}

Gender equality exists in schools without a doubt. The school is not only an important location for the transmission of social culture, but it is also an educational institution that changes gender inequality and leads to the equality. This function of the school is manifested in two ways: on the one hand, in the educational philosophy of the school teacher to change and eliminate all types of gender inequalities in the school; on the other hand, more importantly, in raising awareness of existing gender injustices. Sexual justice should be pursued as a value in school education so that girls recognize and try to understand its flaws. The pursuit of sexual justice necessitates the concerted efforts of men as well. Education teaches boys that society's culture contains far too many prejudices and demands that lead to a willingness to assist women, and that we must insist that these efforts be viewed as processes that help us achieve justice. Only by recognizing their place and role in this regard, as well as eliminating gender bias, can teachers enable their students to pursue sexual justice at all times.

\section{References}

[1] Sun Q, Xu Fuli. Gender inequity in primary school education and countermeasures [J]. Chinese and foreign entrepreneurs, 2019, No. 661(35):162-164.

[2] Sun Qi, Xu Fuli. Gender Inequity in Primary School Education and Countermeasures[J]. Chinese and foreign entrepreneurs, 2019 (35).

[3] Zhang Xue-Min, Wu Zhen-Hua. Multidimensional Measurement and Comparison of Gender Equity in Education [J]. Education and Economics, 2019, 147(01):18-26. 